\title{
A NOTE ON CONVERGENCE IN LENGTH
}

\author{
MIRIAM C. AYER AND TIBOR RADÓ
}

1. Introduction. Let $I$ be a closed linear interval $a_{0} \leqq t \leqq b_{0}$. Let $\mathfrak{r}(t)=(x(t), y(t), z(t)), t \in I$, represent a vector function whose three components $x(t), y(t), z(t)$ are of bounded variation and continuous on $I$. This vector function determines in Euclidean 3-space a curve $x=x(t), y=y(t), z=z(t)$ whose length we denote by $L(\mathfrak{x})$. By convergence in length of a sequence of such vector functions $\mathfrak{x}_{n}(t)=\left(x_{n}(t)\right.$, $\left.y_{n}(t), z_{n}(t)\right), n=0,1,2, \cdots$, is meant that $x_{n}(t), y_{n}(t), z_{n}(t)$ converge uniformly on $I$ to $x_{0}(t), y_{0}(t), z_{0}(t)$ respectively and that $L\left(\mathfrak{x}_{n}\right)$ converges to $L\left(\mathfrak{x}_{0}\right)$. We denote by $V(f)$ the total variation on $I$ of a scalar function $f(t)$ which is continuous and of bounded variation on $I$. By convergence in variation of a sequence $f_{n}(t), n=0,1, \ldots$, is meant that $f_{n}(t)$ is continuous and of bounded variation on $I$ for $n=0,1, \cdots$, that $f_{n}(t)$ converges uniformly on $I$ to $f_{0}(t)$, and that $V\left(f_{n}\right) \rightarrow V\left(f_{0}\right)$. These concepts are due to Adams, Clarkson, and Lewy $[1,2] .^{1}$

We are concerned here with the problem of determining conditions under which convergence in length holds. Uniform convergence on $I$ of the components $x_{n}(t), y_{n}(t), z_{n}(t)$ to $x_{0}(t), y_{0}(t), z_{0}(t)$ respectively implies only that $\lim$ inf $L\left(\mathfrak{x}_{n}\right) \geqq L\left(\mathfrak{x}_{0}\right)$. It is also well known (see $[2,4,5])$ that convergence in length of such a sequence $\mathfrak{x}_{n}$ implies convergence in variation of each of the three sequences of components-and, indeed, convergence in variation of any sequence of scalar functions obtained by projecting the curves $\mathfrak{x}=\mathfrak{x}_{n}(t), t \in I$, $n=0,1, \cdots$, on any line whatever. As a consequence of this we see that convergence in length of the sequence $\mathfrak{x}_{n}(t)$ implies convergence in variation of the sequence $c_{1} x_{n}(t)+c_{2} y_{n}(t)+c_{3} z_{n}(t)$ for arbitrary choice of the constants $c_{1}, c_{2}, c_{3}$. Convergence in variation of each of the three sequences of components is not sufficient to ensure convergence in length of the sequence of vectors (see [2]). In connection with the work of A. P. Morse [4] there arose the question as to whether convergence in length is implied by convergence in variation of every linear combination of the components. This has already been proved by Morse [4] for the case where $\mathfrak{x}_{n}(t)$ is of the special form $\left(t, y_{n}(t), 0\right), n=0,1, \ldots$ In this note we generalize Morse's result to the parametric case. The proof is based on a generalization, 1947.

Presented to the Society, September 4, 1947; received by the editors August 13,

${ }^{1}$ Numbers in brackets refer to the bibliography at the end of the paper. 
due essentially to Steinhaus [6], of a remarkable formula for arc length devised by Cauchy [3].

2. Preliminaries. If each component of a vector function $\mathfrak{x}(t)=(x(t)$, $y(t), z(t)), t \in I$, is BV (of bounded variation), then $\mathfrak{x}(t)$ is said to be $\mathrm{BV}$; if each component is AC (absolutely continuous), then $\mathfrak{x}(t)$ is said to be AC; if each component is continuous, then $\mathfrak{x}(t)$ is said to be continuous. We denote by $\mathfrak{x}^{\prime}(t)$ the vector $\left(x^{\prime}(t), y^{\prime}(t), z^{\prime}(t)\right)$ wherever the derivatives $x^{\prime}(t), y^{\prime}(t), z^{\prime}(t)$ all exist. If the components $x_{n}(t)$, $y_{n}(t), z_{n}(t)$ of a sequence of vectors $\mathfrak{x}_{n}(t), t \in I, n=0,1, \cdots$, converge uniformly on $I$ to $x_{0}(t), y_{0}(t), z_{0}(t)$ respectively, we say that $\mathfrak{x}_{n}(t)$ converges uniformly on $I$ to $\mathfrak{x}_{0}(t)$.

Let $\Delta$ denote any subinterval $t^{\prime} \leqq t \leqq t^{\prime \prime}$ contained in $I$ and let $D(I)$ denote any subdivision of $I$ into a finite number of nonoverlapping intervals $\Delta$. The length $L(\mathfrak{x})$ of a vector function $\mathfrak{x}(t), t \in I$, is defined as

$$
L(\mathfrak{x})=\text { l.u.b. } \sum\left|\mathfrak{x}\left(t^{\prime \prime}\right)-\mathfrak{x}\left(t^{\prime}\right)\right|, \quad \Delta \in D(I),
$$

where the least upper bound is taken over all subdivisions $D(I)$.

We note that this definition of length of vector functions is the exact analog of total variation of scalar functions and that it agrees with the usual definition of length of a curve if $\mathfrak{x}(t)$ is BV and continuous on $I$ and we think of $\mathfrak{x}(t)$ as determining a curve $x=x(t)$, $y=y(t), z=z(t), a_{0} \leqq t \leqq b_{0}$.

We mention now the following well known facts which will be used in this note.

(a) If $\mathfrak{x}(t)=(x(t), y(t), z(t))$ is BV and continuous on $I$, then

$$
V(x) \leqq L(\mathfrak{x}) \leqq V(x)+V(y)+V(z) .
$$

(b) If $f_{n} \rightarrow f_{0}(V)$, then $k f_{n} \rightarrow k f_{0}(V)$ for arbitrary choice of the constant $k$.

(c) If $f_{n}(t)$ converges uniformly on $I$ to $f_{0}(t)$ and if $f_{n}(t)$ is BV and continuous on $I$ for all $n$, then $\lim \inf V\left(f_{n}\right) \geqq V\left(f_{0}\right)$.

(d) If $f(t), t \in I$, is BV and continuous, then $f^{\prime}(t)$ is summable on $I$ and $V(f) \geqq \int_{I}\left|f^{\prime}\right| d t$, the sign of equality holding if and only if $f(t)$ is AC.

(e) If $\mathfrak{x}(t)$ is $\mathrm{BV}$ and continuous on $I$ and if $\mathfrak{x}=\mathfrak{x}_{n}(t), t \in I$, $n=1,2, \cdots$, is a sequence of polygons inscribed in the curve $\mathfrak{x}=\mathfrak{x}(t), t \in I$, and converging uniformly on $I$ to $\mathfrak{x}=\mathfrak{x}(t), t \in I$, then $\mathfrak{x}_{n} \rightarrow \mathfrak{r}(L)$.

(f) Let $\mathfrak{x}_{n}(t)=\left(x_{n}(t), y_{n}(t), z_{n}(t)\right), t \in I, n=0,1, \ldots$ If $\mathfrak{x}_{n} \rightarrow \mathfrak{x}_{0}(L)$, then $x_{n} \rightarrow x_{0}(V)$. 
(g) If $\mathfrak{x}(t), t \in I$, is $\mathrm{BV}$ and continuous, then $\left|\mathfrak{x}^{\prime}(t)\right|$ is summable on $I$ and $L(\mathfrak{x}) \geqq \int_{I}\left|\mathfrak{x}^{\prime}\right| d t$, the sign of equality holding if and only if $\mathfrak{x}(t)$ is $\mathrm{AC}$.

Lemma. Suppose $\mathfrak{x}_{n} \rightarrow \mathfrak{r}_{0}(L)$. Let $\mathfrak{u}$ be any fixed unit vector and $f_{n}(t)$ the scalar product of the vectors $\mathfrak{x}_{n}(t)$ and $\mathfrak{u}$; that is,

$$
f_{n}(t)=\mathfrak{x}_{n}(t) \cdot \mathfrak{u}, \quad t \in I, n=0,1, \cdots .
$$

Then $f_{n} \rightarrow f_{0}(V)$ and $V\left(f_{n}\right) \leqq L\left(\mathfrak{x}_{n}\right)$ for $n=0,1, \cdots$.

The function $f_{n}(t)$ defined here is the projection of the curve $\mathfrak{x}=\mathfrak{x}_{n}(t)$ on a line parallel to the given vector $\mathfrak{u}$. This fact is well known, as was mentioned in $\$ 1$, but a proof will be included for the convenience of the reader. Let $\mathfrak{u}$ be the vector $(a, b, c), a^{2}+b^{2}+c^{2}=1$. Then $f_{n}(t)$ $=a x_{n}(t)+b y_{n}(t)+c z_{n}(t), n=0,1, \cdots$. Let us set up a new system of rectangular coordinates $x^{*}, y^{*}, z^{*}$, such that the $x^{*}$-axis coincides with the line through the origin with direction cosines $a, b, c$. Then $x^{*}$ is expressed in terms of the old coordinates as $a x+b y+c z$. Let $\mathfrak{x}_{n}^{*}(t)=\left(x_{n}^{*}(t), y_{n}^{*}(t), z_{n}^{*}(t)\right)$ denote the vector $\mathfrak{x}_{n}(t)$ referred to the new coordinates. Since arc length is independent of the particular coordinate system used, we have $\mathfrak{x}_{n}{ }^{*} \rightarrow \mathfrak{r}_{0}{ }^{*}(L)$ and hence (see (f), (a)) $x_{n}^{*} \rightarrow x_{0}^{*}(V)$ and $V\left(x_{n}^{*}\right) \leqq L\left(\mathfrak{x}_{n}^{*}\right)$. But $x_{n}^{*}(t)=a x_{n}(t)+b y_{n}(t)+c z_{n}(t)$ $=f_{n}(t), t \in I, n=0,1, \cdots$. That is,

$$
f_{n} \rightarrow f_{0}(V) \text { and } V\left(f_{n}\right) \leqq L\left(\mathfrak{x}_{n}\right)
$$

for all $n$.

3. Cauchy's formula. The formula for arc length which is stated in Lemma 4 applies to any continuous, rectifiable curve in Euclidean 3 -space. It is a direct generalization of a formula of Cauchy [3], the method of proof given here being due to Steinhaus [6]. A proof of the formula is included in this note because the reasoning involved in it is used in $\S 4$, as well as the formula itself.

LEMMA 1. Let $\mathfrak{a}$ be a fixed vector and $\mathfrak{u} a$ variable unit vector from the center of a unit sphere to any point $p$ on the surface $S$ of the sphere. Then

$$
\iint_{S}|\mathfrak{a} \cdot \mathfrak{u}| d \sigma=2 \pi|\mathfrak{a}|,
$$

where $d \sigma$ is the area-element on $S$.

PROof. Choose rectangular coordinates so that the $z$-axis coincides in direction with $a$. In terms of the spherical coordinates $1, \theta, \phi$ of the point $p$ on $S$, we have $\mathfrak{a} \cdot \mathfrak{u}=|\mathfrak{a}| \cdot \cos \phi$ and hence 


$$
\begin{aligned}
\iint_{S}|\mathfrak{a} \cdot \mathfrak{u}| d \sigma & =\int_{0}^{\pi} \int_{0}^{2 \pi}|\mathfrak{a}| \cdot|\cos \phi| \sin \phi d \theta d \phi \\
& =2 \pi|\mathfrak{a}| \int_{0}^{\pi}|\cos \phi| \sin \phi d \phi \\
& =2 \pi|\mathfrak{a}| \int_{0}^{\pi / 2} \sin 2 \phi d \phi=2 \pi|\mathfrak{a}|
\end{aligned}
$$

LEMMA 2. Let $\mathfrak{x}(t)$ be $B V$ and continuous on $I$. Let $S$ be the surface of a unit sphere, $p$ any point on $S$, and $\mathfrak{u}$ the vector from the center of the sphere to $p$. For each point $p$ on $S$ let $V(p)$ denote the total variation of the function $f(t, p)=\mathfrak{x}(t) \cdot \mathfrak{u}, t \in I$. Then $V(p)$ is summable on the surface $S$.

Proof. Since $f(t, p)$ is clearly BV and continuous on $I$ for each point $p$ on $S, V(p)$ is defined for every $p$. Consider now a sequence of points $p_{n}$ on $S$ such that $p_{n} \rightarrow p_{0}$. It is easily verified that the sequence $f\left(t, p_{n}\right)$ converges uniformly on $I$ to $f\left(t, p_{0}\right)$, from which it follows that $\lim$ inf $V\left(p_{n}\right) \geqq V\left(p_{0}\right)$ (see (c) of $\$ 2$ ). This means that $V(p)$ is lower semi-continuous on $S$ and hence measureable on $S$. From the fact that $V(p)$ is bounded on $S$ by $L(\mathfrak{x}$ ) (see Lemma of $\S 2$ ), we conclude that $V(p)$ is summable on $S$.

LEMMA 3. Given a sequence of $B V$, continuous vector functions $\mathfrak{x}_{n}(t)$, $t \in I, n=0,1, \cdots$. Let $S, p, \mathfrak{u}$ be defined as in Lemma 2. For each $p$ on $S$ let $V_{n}(p)$ denote the total variation of the function $f_{n}(t, p)=\mathfrak{x}_{n}(t) \cdot \mathfrak{u}$, $t \in I, n=0,1, \cdots$. If the sequence $f_{n}(t, p)$ converges in variation for every point $p$ on $S$, then

$$
\iint_{S} V_{n}(p) d \sigma \rightarrow \iint_{S} V_{0}(p) d \sigma
$$

where $d \sigma$ is the area-element on $S$.

Proof. By hypothesis the sequence $f_{n}(t, p)$ converges in variation for every point $p$ on $S$. Hence

$$
V_{n}(p) \rightarrow V_{0}(p), \quad p \in S .
$$

Let $a, b, c$ be any three (real) constants such that $a^{2}+b^{2}+c^{2}=1$. Since the point $(a, b, c)$ lies on $S$, we then have by (1) convergence in variation of the sequence $a x_{n}(t)+b y_{n}(t)+c z_{n}(t)$ and therefore

$$
V\left(a x_{n}+b y_{n}+c z_{n}\right) \rightarrow V\left(a x_{0}+b y_{0}+c z_{0}\right) \text {. }
$$

In particular, 


$$
V\left(x_{n}\right) \rightarrow V\left(x_{0}\right), \quad V\left(y_{n}\right) \rightarrow V\left(y_{0}\right), \quad V\left(z_{n}\right) \rightarrow V\left(z_{0}\right) .
$$

Summability of each function $V_{n}(p)$ follows from Lemma $2 ; V_{n}(p)$ converges on $S$ to $V_{0}(p)$ by (1). In order to prove convergence of their integrals it will therefore be sufficient to show that the sequence $V_{n}(p)$ is bounded on $S$. For each point $p$ on $S$ we have by the Lemma 1 and (a) of $\$ 2$

$$
V_{n}(p) \leqq L\left(\mathfrak{x}_{n}\right) \leqq V\left(x_{n}\right)+V\left(y_{n}\right)+V\left(z_{n}\right), \quad n=0,1, \cdots .
$$

But (2) implies the existence of a constant $M$ such that

$$
V\left(x_{n}\right) \leqq M, \quad V\left(y_{n}\right) \leqq M, \quad V\left(z_{n}\right) \leqq M, \quad n=0,1, \cdots .
$$

Inequalities (3) and (4) establish the fact that the sequence $V_{n}(p)$ is bounded on $S$ and hence, as remarked above,

$$
\iint_{S} V_{n}(p) d \sigma \rightarrow \iint_{S} V_{0}(p) d \sigma .
$$

LEMMA 4. Under the hypotheses of Lerma 2,

$$
L(\mathfrak{x})=(2 \pi)^{-1} \iint_{S} V(p) d \sigma,
$$

where $d \sigma$ is the area-element on $S$ (this is the generalized Cauchy formula, see §3).

Proof. Let us suppose first that $\mathfrak{x}(t)$ is AC. It is clear that for fixed $p$ the function $f(t, p)$ is $\mathrm{AC}$ on $I$ and that its derivative is equal to $\mathfrak{x}^{\prime}(t) \cdot \mathfrak{u}$ except on a subset of $I$ of measure zero. Hence (by (d) of §2)

$$
V(p)=\int_{I}\left|\mathfrak{r}^{\prime}(t) \cdot \mathfrak{u}\right| d t .
$$

But absolute continuity of $\mathfrak{x}(t)$ also implies (see $(\mathrm{g})$ of $\S 2$ )

$$
L(\mathfrak{x})=\int_{I}\left|\mathfrak{x}^{\prime}(t)\right| d t .
$$

Since $V(p)$ is summable on $S$ (see Lemma 2), we obtain by use of (1)

$$
\begin{aligned}
\iint_{S} V(p) d \sigma & =\iint_{S}\left[\int_{I}\left|\mathfrak{x}^{\prime}(t) \cdot \mathfrak{u}\right| d t\right] d \sigma \\
& =\int\left[\iint_{S}\left|\mathfrak{x}^{\prime}(t) \cdot \mathfrak{u}\right| d \sigma\right] d t,
\end{aligned}
$$


where the theorem of Tonelli justifies the changes in the order of integration. From (3), Lemma 1, and (2) we conclude that

$$
\begin{aligned}
(2 \pi)^{-1} \iint_{S} V(p) d \sigma & =(2 \pi)^{-1} \int_{I}\left[\iint_{S}\left|\mathfrak{x}^{\prime}(t) \cdot \mathfrak{u}\right| d \sigma\right] d t \\
& =(2 \pi)^{-1} \int_{I}(2 \pi)\left|\mathfrak{x}^{\prime}(t)\right| d t=L(\mathfrak{x}) .
\end{aligned}
$$

Let us suppose next that $\mathfrak{x}(t)$ is merely BV and continuous on $I$. Define $\mathfrak{x}=\mathfrak{x}_{n}(t), t \in I, n=1,2, \cdots$, to be a sequence of polygons inscribed in the curve $\mathfrak{x}=\mathfrak{x}(t), t \in I, \mathfrak{x}_{n}(t)$ converging uniformly on $I$ to $\mathfrak{x}(t)$. By (e) of $\$ 2$ we then have $\mathfrak{x}_{n} \rightarrow \mathfrak{x}_{0}(L)$ and hence

$$
L\left(\mathfrak{x}_{n}\right) \rightarrow L(\mathfrak{x}) .
$$

By the lemma of $\$ 2$ we also have convergence in variation of the sequence $f_{n}(t, p)=\mathfrak{x}_{n}(t) \cdot \mathfrak{u}, t \in I$, for every point $p$ on $S$. Application of Lemma 3 then yields the result that

$$
\iint_{S} V_{n}(p) d \sigma \rightarrow \iint_{S} V(p) d \sigma
$$

where $V_{n}(p)$ is defined as in Lemma 3 for $n=1,2, \cdots$. But since each approximating function $\mathfrak{x}_{n}(t)$ is $\mathrm{AC}$, we can express its length in the form

$$
L\left(\mathfrak{x}_{n}\right)=(2 \pi)^{-1} \iint_{S} V_{n}(p) d \sigma, \quad n=1,2, \cdots .
$$

In conjunction with (4) and (5) this implies

$$
L(\mathfrak{x})=\lim L\left(\mathfrak{x}_{n}\right)=\lim (2 \pi)^{-1} \iint_{S} V_{n}(p) d \sigma=(2 \pi)^{-1} \iint_{S} V(p) d \sigma .
$$

4. The theorem. Let $\mathfrak{x}_{n}(t)=\left(x_{n}(t), y_{n}(t), z_{n}(t)\right), n=0,1, \cdots$, be a sequence of vectors which are BV and continuous on $I$. Then $\mathfrak{x}_{n} \rightarrow \mathfrak{x}_{0}(L)$ if and only if the sequence $c_{1} x_{n}(t)+c_{2} y_{n}(t)+c_{3} z_{n}(t)$ converges in variation for every choice of the (real) constants $c_{1}, c_{2}, c_{3}$.

Proof. Sufficiency. By hypothesis the sequence $c_{1} x_{n}(t)+c_{2} y_{n}(t)$ $+c_{3} z_{n}(t)$ converges in variation for every choice of the constants $c_{1}, c_{2}, c_{3}$. This implies uniform convergence of $\mathfrak{x}_{n}(t)$ to $\mathfrak{x}_{0}(t)$ and also convergence in variation of $f_{n}(t, p)$ for every point $p$ on $S$, where $f_{n}(t, p)$ is defined as in Lemma 3. From Lemmas 3 and 4 we then conclude that 


$$
\lim L\left(\mathfrak{x}_{n}\right)=\lim (2 \pi)^{-1} \iint_{S} V_{n}(p) d \sigma=(2 \pi)^{-1} \iint_{S} V_{0}(p) d \sigma=L\left(\mathfrak{x}_{0}\right)
$$

and hence $\mathfrak{x}_{n} \rightarrow \mathfrak{x}_{0}(L)$.

Necessity. If $\mathfrak{x}_{n} \rightarrow \mathfrak{x}_{0}(L)$, the sequence $f_{n}(t, p)$ converges in variation for every point $p$ on $S$ (see Lemma of $\$ 2$ ). Let $c_{1}, c_{2}, c_{3}$ be any three (real) constants. If $c_{1}=c_{2}=c_{3}=0$, then the statement is trivial. Otherwise let $\mathfrak{u}$ be a unit vector with direction cosines proportional to $c_{1}, c_{2}, c_{3}$. The desired relation now follows readily from the lemma and $(b)$ of $\$ 2$.

\section{BIBLIOGRAPHY}

1. C. R. Adams and J. A. Clarkson, On convergence in variation, Bull. Amer. Math. Soc. vol. 40 (1934) pp. 413-417.

2. C. R. Adams and H. Lewy, On convergence in length, Duke Math. J. vol. 1 (1935) pp. 19-26.

3. A. Cauchy, Mémoire sur la rectification des courbes et la quadrature des surfaces courbes, Oeuvres complètes, series 1, vol. 2, 1908, pp. 167-177,

4. A. P. Morse, Convergence in variation and related topics, Trans. Amer. Math. Soc. vol. 41 (1937) pp. 48-83.

5. T. Rad6 and $P$. Reichelderfer, Convergence in length and convergence in area, Duke Math. J. vol. 9 (1942) pp. 527-565.

6. H. Steinhaus, Sur la portée pratique et therorique de quelques théorèmes sur la mesure des ensembles de droits, Comptes Rendus du Premier Congrès des Mathematiciens des Pays Slaves, Warsaw, 1929, pp. 348-354.

Wellesley College and The Ohio State University 